\title{
Ongoing Clinical Trials in Testicular Cancer: The TIGER Trial
}

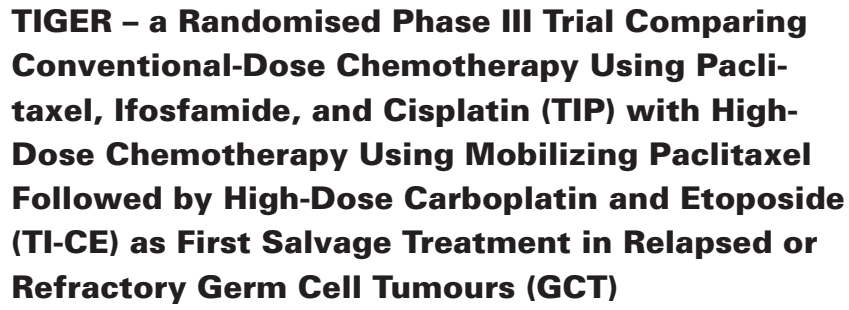

\section{Condition}

Male Relapsed/Refractory Germ Cell Tumor

ClinicalTrials.gov Identifier

NCT02375204

EudraCT Number

2014-003930-17

Study Design

Study Type: Interventional

Study Phase: III

Allocation: Randomized

Intervention Model: Parallel Assignment

Masking: Open-label

Primary Purpose: Treatment

Estimated Enrollment: 420 patients

\section{Intervention}

Drugs: Paclitaxel iv, ifosfamide iv, cisplatin iv, pegylated g-csf sc (Arm A); paclitaxel iv, ifosfamide iv, cisplatin iv, pegylated g-csf sc, carboplatin iv, etoposide phosphate iv (Arm B)
Study Arm A (active comparator): Patients will receive treatment for 4 cycles administered every 21 days with paclitaxel $250 \mathrm{mg} / \mathrm{m}^{2}$ iv over $24 \mathrm{~h}$ on day 1 , ifosfamide $1.500 \mathrm{mg} / \mathrm{m}^{2}$ iv daily on days $2-5$, cisplatin $25 \mathrm{mg} / \mathrm{m}^{2}$ iv daily on days $2-5$, and pegylated G-CSF SC on day 6 or 7 or G-CSF on days 6-18. Study Arm B (experimental): Patients will receive treatment for 5 cycles in total. Cycle 1-2: Paclitaxel $200 \mathrm{mg} / \mathrm{m}^{2}$ iv over $3 \mathrm{~h}$ on day 1 , ifosfamide $2.000 \mathrm{mg} / \mathrm{m}^{2}$ iv on days $1-3$ administered every 14 days, and G-CSF $10 \mu \mathrm{g} / \mathrm{kg}$ sc on days 3-15 (cycle 1) and 3-14 (cycle 2) or pegylated G-CSF $6 \mathrm{mg}$ sc on day 4 or 6 (cycle 1) and day 4 or 5 (cycle 2) with subsequent leukapheresis every 14 days, if there is an inadequate number of CD34+ cells/ $\mathrm{kg}$ collected in cycle 1. Cycle 3-5: Carboplatin AUC8 iv daily on days $1-3$, etoposide $400 \mathrm{mg} / \mathrm{m}^{2}$ iv daily on days $1-3$, autologous stem cell reinfusion on day 5 , and pegylated G-CSF $6 \mathrm{mg} \mathrm{SC}$ or G-CSF $5 \mu \mathrm{g} / \mathrm{kg}$ sc daily on days $5-15$.

Primary Outcome Measure

Overall survival (time frame: up to 36 months post-treatment)

\section{Sponsor}

Alliance for Clinical Trials in Oncology

\section{Co-Sponsor}

National Cancer Institute (NCl), European Organisation for Research and Treatment of Cancer (EORTC), Movember Foundation

\section{Contacts}

Study chair worldwide: Darren Feldman (+1 646422 4491) Study chair Europe: Thomas Powles (+44 207882 8283)

\section{Description}

This international randomized, multicenter open label phase III trial is a collaboration between the US American Alliance for Clinical Trials in Oncology and the European Organisation for Research and Treatment of Cancer (EORTC). Patients will be recruited at selected European, US American, and Australian sites.

Monetary support for trial realization will be provided by the National Cancer Institute (NCI) in the US, the European Organization for Research and Treatment of Cancer (EORTC), and the Movember Foundation in Europe and Australia. In addition several nations will get financial support from country-specific grants.
In France by the PHRC (le programme hospitalier de recherche clinique), in Italy by AIFA (Agenzia Italiana del Farmaco) and in the UK by CRUK (Cancer Research UK) and the Orchid Foundation. For Germany, a grant application at the Deutsche Krebshilfe $(\mathrm{DKH})$ is currently under review.

The trial has been designed to prospectively assess the impact of sequential high-dose chemotherapy given at first relapse compared to conventional-dose standard chemotherapy on patients' overall survival (OS) (primary outcome measure), as well as progressionfree survival (PFS), favorable response rate and clinically relevant parameters such as treatment related toxicity and mortality, impact of tumor marker decline rates on OS and PFS, a prospective eva- 
luation of the IPFSG risk stratification model (secondary outcome measures) and a stratification by region (North America and Europe).

The randomization will be 1:1 (comparator : experimental arm) without cross-over on an intent-to-treat basis. Overall, 420 patients will be recruited, 210 patients per arm. It is anticipated that about 170 patients from the Alliance sites and about 250 patients from the European sites will be enrolled. Germany expects to contribute a minimum of 70-75 patients.

\section{Eligibility Criteria}

\section{Main Inclusion Criteria}

- Male gender

- Age $\geq 18$ years for Germany

- ECOG Performance Status 0-2

- GCT histology (seminoma and nonseminoma)

- Unequivocal progression of measurable disease following one line of cisplatin-based chemotherapy
- Unequivocal progression of non-measurable disease with consecutive elevated markers following one line of cisplatin-based chemotherapy

- A minimum of 3 and maximum of 6 cisplatin-based treatment cycles

- No more than 1 prior line of chemotherapy for GCT

- Patients with late relapses who have unresectable disease

- Completion of a full informed consent

\section{Main Exclusion Criteria}

- More than 1 prior line of chemotherapy for GCT

- Prior treatment with TIP or high-dose chemotherapy

- Concurrent treatment with other cytotoxic drugs or targeted therapies

- Radiation therapy (other than to the brain) within 14 days of day 1 of the protocol

- The existence of a concurrent malignancy

- Patients with resectable late relapses

- Patients with large ( $\geq 2 \mathrm{~cm})$, hemorrhagic, or symptomatic brain metastases 


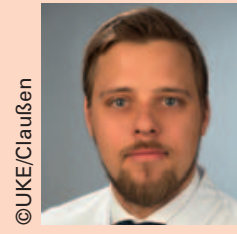

\section{Dr. Christoph Oing}

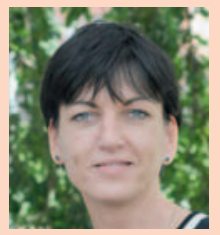

Prof. Dr. Anja Lorch

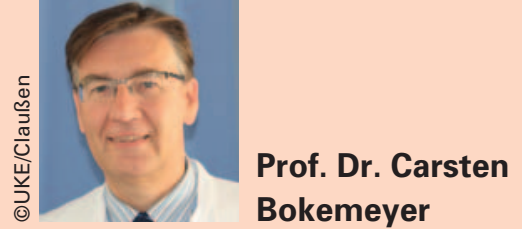

\section{Why this Trial Is Important to Us}

Germ cell tumors are the most common malignancy among men aged between 15 and 45 years [1] and are a model of a curable solid malignancy due to an extraordinary sensitivity towards cisplatin-based combination chemotherapy [2]. Dependent on the extent of dissemination and tumor maker elevation at primary diagnosis, $10-50 \%$ of patients will fail first-line treatment and need salvage treatment [3]. In a large retrospective analysis of 1,594 patients conducted by the International Prognostic Factor Study Group (IPFSG) 6 factors could be identified to stratify patients failing first-line treatment between 5 risk groups with a 3 -year survival probability ranging from 6 to 77\% [4]. The question whether or not immediate intensification of first salvage treatment by sequential high-dose chemotherapy (HDCT) with subsequent autologous stem cell transplantation (ASCT) improves treatment success is part of an ongoing debate.

Nowadays, first salvage treatment with curative intent comprises either 4 cycles of conventional-dose triple combination chemotherapy (CDCT), e.g. cisplatin and ifosfamide with paclitaxel (TIP), etoposide (VIP) or vinblastine (VeIP), or sequential carboplatin-etoposide based high-dose chemotherapy (HDCT) for 2-3 cycles with subsequent autologous peripheral blood stem cell transplantation (for a recent comprehensive review see [5]). The first phase I / II trial evaluating HDCT with 2 cycles carboplatin and etoposide with subsequent autologous bone marrow transplantation in 33 absolutely cisplatin-refractory patients or after failure of at least 2 cisplatin-based CDCT regimens was reported in 1989. Remarkably, complete responses were achieved in $25 \%$ of patients, but there was excessive treatment-related mortality of $21 \%$ [6].

However, due to a lack of randomized trials, the optimal first salvage approach still remains unclear. It is a matter of debate whether sequential high-dose chemotherapy (HDCT) or conventional-dose chemotherapy (CDCT) represents the optimal strategy for patients who progress after first-line chemotherapy. Practices vary throughout the world with some expert centers (Indiana investigators, German investigators) administering high-dose chemotherapy as initial salvage treatment to all patients, whereas others use HDCT only in patients who failed initial salvage therapy with CDCT. Others use a risk stratified approach, with patients presenting with favorable prognostic factors at relapse re- ceiving CDCT and patients presenting with poor prognostic factors being treated with HDCT as initial salvage regimen.

Only 1 prior randomized trial (IT-94 study) attempted to answer this question. No benefit of HDCT over CDCT was shown, however, the trial only used 1 cycle of HDCT, whereas 2 or $3 \mathrm{cy}$ cles of HDCT are considered necessary for optimal benefit and are widely used as standard regimen. In contrast, many other retrospective studies, including the above-mentioned and recently published analysis of 1,594 patients have suggested a benefit in both PFS and OS for HDCT over CDCT as initial salvage chemotherapy.

TIGER will now be able to answer this important and controversial question about optimal treatment for first salvage in relapsed and refractory GCT patients. There are 3 possible outcomes to this trial, all of which would have important effects on the standard of care:

1) HDCT improves OS compared to CDCT in all patient groups.

2) HDCT does not improve OS compared to CDCT in any patient groups.

3) HDCT improves OS relative to CDCT only in a specific subset of patients.

\section{Summary}

Using 1-2 cycles of TI for stem cell mobilization with subsequent harvest of peripheral blood stem cells followed by 2-3 cycles of HD-CE plus ASCT is currently the HDCT approach of choice. As a consequence, TI-CE was chosen as the experimental arm regimen in the TIGER trial. As the comparator, 4 cycles of TIP were selected based on a high and durable progression-free rate of $63 \%$ in a phase II trial [7].

The TIGER trial is an excellent example for an international collaboration trial, which was finally initialized after many years of organizational and monetary obstacles that had to be overcome. Major funding by the NCI, the EORTC, the Movember Foundation, and several additional national grants made this study possible. Currently the trial is recruiting patients in the US and will soon start to recruit patients in Europe. First results of this groundbreaking trial can be expected in a few years only, but there is no other way to resolve the uncertainty regarding the role of HDCT as treatment at first relapse. 


\section{References}

1 Ruf CG, et al.: Changes in epidemiologic features of testicular germ cell cancer: age at diagnosis and relative frequency of seminoma are constantly and significantly increasing. Urol Oncol 2014;32:33 e1-6.

2 Einhorn LH: Treatment of testicular cancer: a new and improved model. J Clin Oncol 1990;8:1777-1781.

3 International Germ Cell Cancer Collaborative Group: International Germ Cell Consensus Classification: a prognostic factor-based staging system for metastatic germ cell cancers. J Clin Oncol 1997;15: 594-603.

4 International Prognostic Factors Study Group: Prognostic factors in patients with metastatic germ cell tumors who experienced treatment failure with cisplatin-based first-line chemotherapy. J Clin Oncol 2010;28:4906-4911.

5 Oing C, et al.: Pharmacotherapeutic treatment of germ cell tumors: standard of care and recent developments. Expert Opin Pharmacother 2016;17:545-560.
6 Nichols CR, et al.: Dose-intensive chemotherapy in refractory germ cell cancer - a phase I/II trial of high-dose carboplatin and etoposide with autologous bone marrow transplantation. J Clin Oncol 1989;7:932-939.

Kondagunta GV, et al.: Combination of paclitaxel, ifosfamide, and cisplatin is an effective second-line therapy for patients with relapsed testicular germ cell tumors. J Clin Oncol 2005;23:6549-6555.

\section{Contact address:}

Commentary Christoph Oing, Anja Lorch, Carsten Bokemeyer Prof. Dr. Anja Lorch

Department of Urology, Genitourinary Medical Oncology

University Hospital Düsseldorf

Moorenstraße 5, 40225 Düsseldorf, Germany

anja.lorch@med.uni-duesseldorf.de 